\title{
Finite Element Analysis of Space Science Experimental Equipment Based on HyperMesh and Nastran
}

\author{
Huang Sinian ${ }^{1,2}$, Wang Anping ${ }^{2}$ \\ ${ }^{1}$ University of Chinese Academy of Science, Beijing 100049, China \\ ${ }^{2}$ Technology and Engineering Center for Space Utilization, Chinese Academy of Science, Beijing 100094, China
}

\begin{abstract}
In the design process of space science experimental equipment, in order to better grasp the dynamic characteristics of the structure, a large number of finite element analysis is needed. This paper comprehensively use HyperMesh and Nastran to establish a set of finite element analysis process for space science experimental equipment, combine the advantages of each software to perform finite element analysis comprehensively, which greatly improves the accuracy and efficiency of the finite element analysis, the problems of this kind of engineering analysis has certain reference value.
\end{abstract}

\section{Introduction}

With the further development of the manned space engineering, the research prospects for the application of space science in China have been presented to our eyes ${ }^{[1]}$. Space science experimental equipment is an important structure for the installation of payloads in spacecraft capsules and is an important part of space application system. In the design process of space science experimental equipment, a large number of finite element simulation analysis are required, through finite element analysis, the designed space science experimental equipment can be optimized based on the analysis results, shortening the development cycle and saving costs ${ }^{[2,3]}$.

Modal analysis is the most important one in the finite element analysis process of the space science experimental equipment, it often generates violent vibration during the spacecraft launching process, which has a huge impact on the space science experimental equipment in the spacecraft. Therefore, how to calculate the inherent characteristics of the system by determining the reference frequency of the science experimental equipment and to avoid resonance in the emission process is necessary. This paper comprehensively use the different characteristics of Creo, HyperMesh and Nastran to establish a set of finite element analysis procedures, the modal analysis of the space science experimental equipment obtains its natural frequency and mode shape, which has certain reference significance.

\section{Comprehensive Application of Nastran and HyperMesh}

For a simple geometric model, generally with a single CAE software can complete the analysis process, however, when performing finite element modeling analysis of a complex model, various software is often comprehensively used to complement each other, and the advantages of each software are combined to perform finite element simulation analysis, it can quickly and efficiently solves engineering problems.

\subsection{HyperMesh Features}

HyperMesh is a finite element pre-processing software developed by Altair Corporation of the United States, it has powerful finite element pre-processing function and can provide users with high-quality and high-efficiency mesh division technology, it can complete pole beam, plate shell and solid mesh division, through the interactive operating environment helps users create various complex finite element models. HyperMesh provides powerful geometric cleanup capabilities that support a variety of automated or manual geometric cleanup and repairs, such as surface stitching, surface repairs and removal of chamfered round holes. HyperMesh supports various mainstream CAD and CAE software currently on the market, it can read different types of geometric model files and can also output finite element model files for different solvers.

HyperMesh is powerful in grid processing, however there are fewer types of materials and units in the finite element analysis, and the solution method is more complex, there is also a certain gap between the finite element analysis results and post-processing ${ }^{[4]}$.

\subsection{Nastran Features}

Nastran is a series of products of the United States MSC.Software Corporation, has been widely used in 
engineering simulation analysis of various industries, MSC.Software products cover almost all research institutions, design departments, universities and other institutions of China Aerospace ${ }^{[5]}$. Nastran has a variety of material libray with a variety of linear and other nonlinear models, which have the advantages of high accuracy and fast convergence in analytical. Figure 1 show a general flow chart for Nastran modeling analysis.

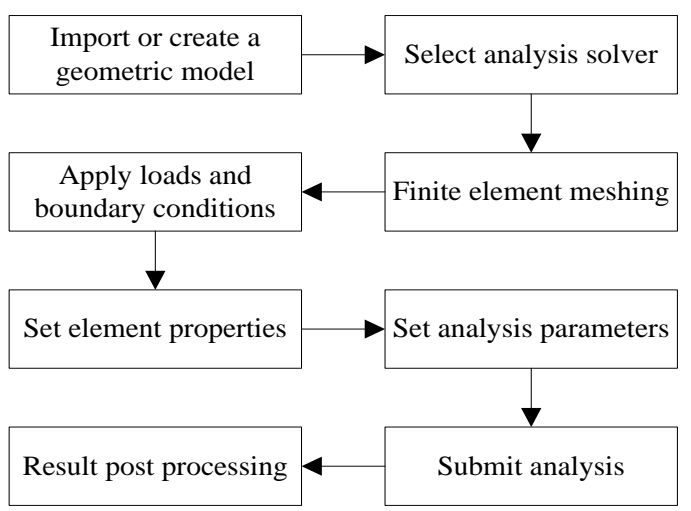

Figure 1. Nastran modelling and analysis flow chart.

Compared with other finite element analysis software, Nastran's meshing capabilities and geometric cleanup capabilities need to be further strengthened.

\subsection{Comprehensive use of Creo, HyperMesh and Nastran Analysis process}

HyperMesh and Nastran have their own advantages when perform finite element analysis, therefore, it is possible to comprehensively utilize the features of each software, complement each other, establish a set of geometric models using Creo, HyperMesh and Nastran to perform finite element analysis, which greatly improve the efficiency of finite element analysis ${ }^{[6]}$. The geometric model created by Creo was imported into HyperMesh, and the steps of geometric model cleaning, mesh division, material attribute assignment, unit attribute assignment and boundary load setting in the finite element analysis process were completed in turn, completing the establishment of the finite element model. Then the finite element model was submitted to Nastran for solving calculations, completing the finite element analysis process. As shown in Figure 2, it is a finite element simulation flow chart.

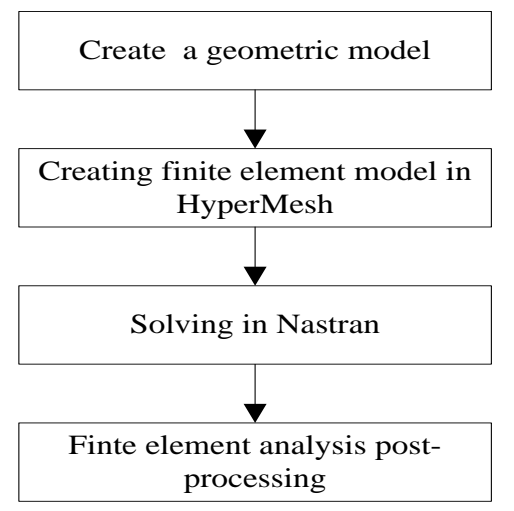

Figure 2. Finite element simulation flow chart.

\section{Modal Analysis of Space Science Experimental Equipment Based on HyperMesh and Nastran}

\subsection{Space Science Experimental Equipment Modal Analysis Theory}

The finite element modal of space science experimental equipment in this paper belongs to the multi-freedom undamped free vibration system ${ }^{[7]}$, the free vibration equation of the undamped system is as follows

$$
\boldsymbol{M} \ddot{\boldsymbol{y}}+\boldsymbol{K y}=0
$$

In the formula, $\boldsymbol{M}$ is the mass matrix, $\boldsymbol{K}$ is the stiffness matrix, $\ddot{y}$ and $\boldsymbol{y}$ are the acceleration vector and the displacement vector respectively, the general solution of (1) cab be expressed as the sum of each special solution, and one set of special solutions is as follows

$$
\boldsymbol{y}=\boldsymbol{A} \sin (\omega t+\varphi)
$$

This is equivalent to assuming that each mass body performs a simple harmonic motion and that the vibration frequency is the same as the initial phase angle, and differentiated twice for $t$ in (2), the following results are obtained:

$$
\ddot{\boldsymbol{y}}=-\omega^{2} \boldsymbol{A} \sin (\omega t+\varphi)
$$

Substituting (2) and (3) into (1) can be obtained

$$
\left(\boldsymbol{K}-\omega^{2} \boldsymbol{M}\right) \boldsymbol{A}=\boldsymbol{O}
$$

So the system's characteristic equation is

$$
\left|\boldsymbol{K}-\omega^{2} \boldsymbol{M}\right|=\boldsymbol{0}
$$

The solution obtains $n$ positive real roots of $\omega_{i}$, finding $n$ frequencies $\omega_{i},(i=1,2,3 \ldots, n)$, where the lowest frequency $\omega_{i}$ is the lowest natural frequency, which is the basic frequency of the system, the remaining frequencies are collectively referred to as high-order frequencies.

\subsection{Establishment of Finite Element Model of Space Science Experimental Equipment}

Space science experimental equipment are generally relatively regular plate structures, which are mainly composed of external frames and internal electronic components and pipelines. In the finite element modeling analysis, the surface is generally treated as a shell element, different thicknesses are assigned to different parts, and reinforcing ribs and mass points are set in the corresponding positions to better simulate the trueness of the structures. In the process of processing and manufacturing, in order to reduce the quality while ensuring the structural strength, the commonly used materials is aluminum alloy. 
Taking the science experimental equipment shown in Fig. 3 as an example, the geometric model created by Creo was imported into HyperMesh, and choosed the Nastran as the solver template to establish the finite element model. In the finite element meshing, the main unit used is four-node shell element and tetrahedron element, the former mainly simulates the structural PCB structure and the latter is mainly used for simulating the external panel, the connection of each part is implemented by the rigid element RBE2. As shown in Fig.4, the structure finite element model established by HyperMesh has a total of 163,000 finite element nodes.

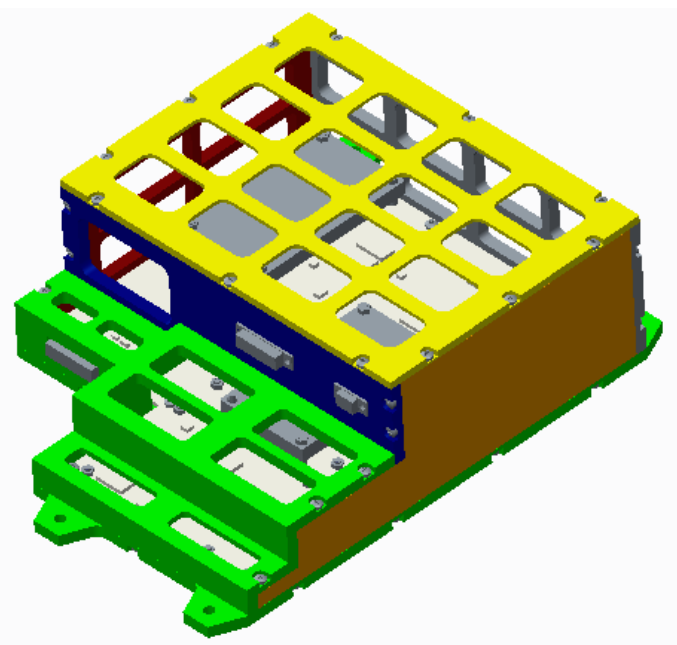

Figure 3. Geometric model of the space science experimental equipment .

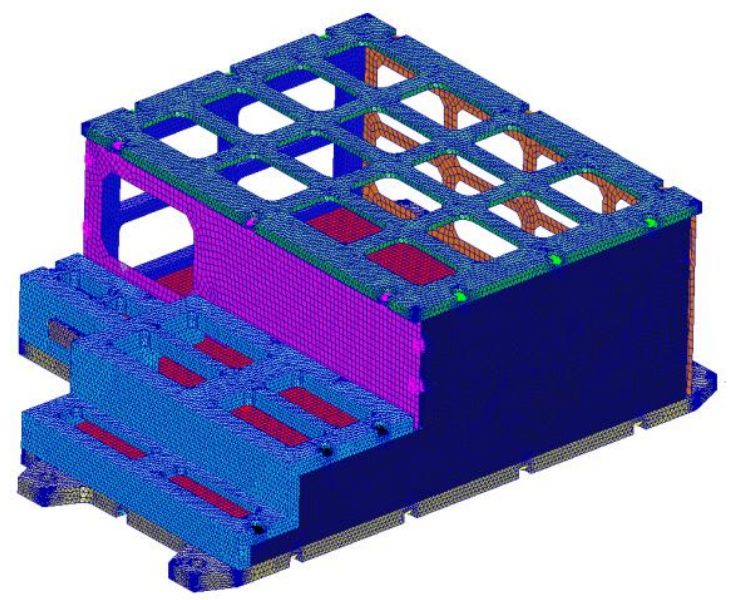

Figure 4. Finite element model of space science experimental equipment.

The experimental equipment is mainly composed of aluminum alloy and internal PCB structure, when defining the material properties, it is necessary to define two kinds of materials at the same time, and set parameters such as desity, Poisson's ratio and elastic modulus of different materials, the material properties of different materials are shown in Table 1.

After the assignment of the matertial properties, the assignment of the unit attributes is performed according to the actual conditions, the thicknesses of the simplified shell units are given different thicknesses, and the corresponding entity attributes are selected for the body units. As the same time, in order to better simulate the real situation of the equipment, different mass points are set on the surface of the equipment. In general, the space science experimental equipment is fixed inside the spacecraft. According to the actual installation form, the four lug positions of the structure are fixed during the analysis. Set the solution parameters in HyperMesh, complete the establishment of the finite element model, and output the finite element model to Nastran for solution calculation.

Table 1. Materials properties.

\begin{tabular}{|c|c|c|c|}
\hline Materials & $\begin{array}{c}\text { Density } \\
\left(\mathrm{kg} \cdot \mathrm{m}^{-3}\right)\end{array}$ & $\begin{array}{c}\text { Poisson's } \\
\text { ratio }\end{array}$ & $\begin{array}{c}\text { Elastic } \\
\text { Modulus }\end{array}$ \\
\hline $\begin{array}{l}\text { Aluminu } \\
\text { m alloy }\end{array}$ & 2700 & 0.33 & $7 \times 10^{10}$ \\
\hline PCB & 1700 & 0.30 & $1.4 \times 10^{10}$ \\
\hline
\end{tabular}

\subsection{Modal Analysis Based on Nastran}

The natural frequency and mode shape of the space experimental equipment are determined by the geometry of the structure, material properties and constraints. In the modal analysis of the structure, the Lanczos Method with a fast convergence rate is selected for analysis, in general, during the modal analysis, it is necessary to understand the local modality and the overall modality of the structure, so it is necessary to find the overall structure starting frequency. In the calculation process, the first 20 natural frequencies of the structure are extracted, and the overall starting frequency of the structure is found through analysis. As shown in Table 2, it is the first 8th order modal frequency and mode result of the structure and the 17th order overall start-up of the structure frequency.

Table 2. Modal analysis results.

\begin{tabular}{|c|c|c|}
\hline Order & $\begin{array}{c}\text { Frequency } \\
(\mathrm{Hz})\end{array}$ & \multicolumn{1}{|c|}{ Mode description } \\
\hline 1 & 167.7 & $\begin{array}{l}\text { the vibration of the PCB and } \\
\text { internal electronic components }\end{array}$ \\
\hline 2 & 170.8 & $\begin{array}{l}\text { the vibration of the PCB and } \\
\text { internal electronic components }\end{array}$ \\
\hline 3 & 221.7 & $\begin{array}{l}\text { the vibration of the PCB and } \\
\text { internal electronic components }\end{array}$ \\
\hline 4 & 258.2 & $\begin{array}{l}\text { the vibration of the PCB and } \\
\text { internal electronic components }\end{array}$ \\
\hline 5 & 272.5 & $\begin{array}{l}\text { the vibration of the PCB and } \\
\text { internal electronic components }\end{array}$ \\
\hline 7 & 319.2 & $\begin{array}{l}\text { the vibration of the PCB and } \\
\text { internal electronic components }\end{array}$ \\
\hline 8 & 347.4 & $\begin{array}{l}\text { the vibration of the PCB and } \\
\text { internal electronic components }\end{array}$ \\
\hline 17 & 567.3 & $\begin{array}{l}\text { the vibration of the PCB and } \\
\text { internal electronic components } \\
\text { external structure }\end{array}$ \\
\hline
\end{tabular}

Select the representative first-order and second-order local vibrations and the 17 th-order global vibration, the modal shapes are shown in Figs. 5-7. 


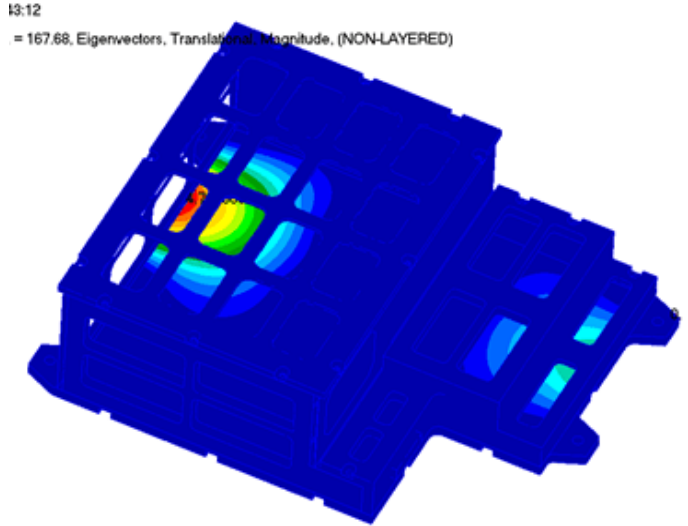

Figure 5. First-order mode shape.

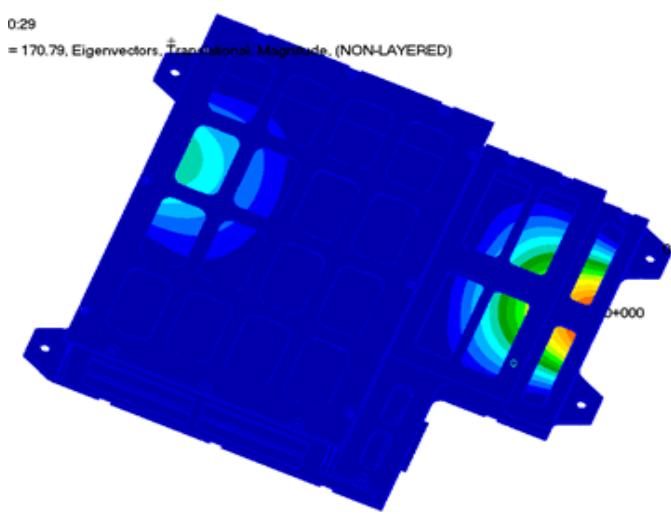

Figure 6. Second-order mode shape.

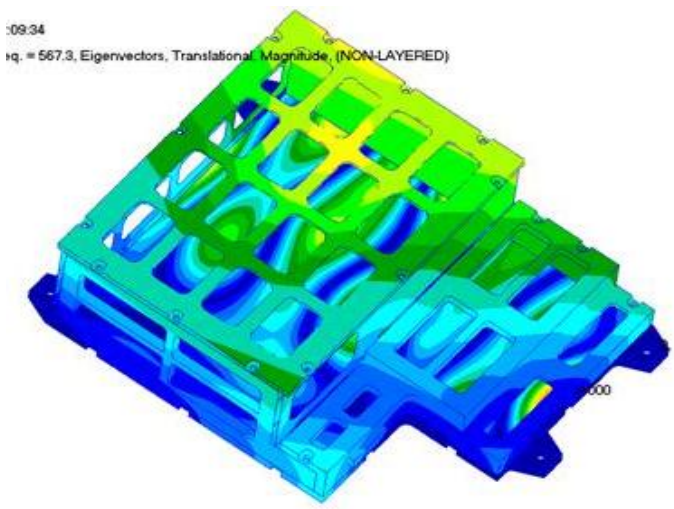

Figure 7. 17th-order mode shape.

From the modal shape diagram and the modal frequency, the basic frequency of the space science experimental equipment is $167.7 \mathrm{~Hz}$, which is the vibration of the PCB and internal electronic components, it is a local vibration mode; the first-order overall modal frequency of the structure is $567.3 \mathrm{~Hz}$. The results have certain reference value for structural design and optimization.

\section{Concluding Remarks}

In this paper, taking the typical structure of the space science experimental equipment as an example, a set of procedures for finite element analysis using Creo 3D modeling software, HyperMesh meshing software and finite element solution software Nastran integrated finite element analysis was established, taking full account of the advantages and disadvantages of each software, giving full play to the advantages of each software, the efficiency and accuracy of finite element analysis have been greatly improved. Through the finite element analysis of the space science experimental equipment, the basic frequency and the overall starting frequency of the structure are found, which can be used as a reference in the design process to avoid resonance. The finite element analysis process has certain reference value and application value for engineering applications.

\section{References}

1. Wang Anping. Modal Analysis and Model Optimization of Space Science Experimental Equipment[J]. ENGINEERING \& TEST, 2017, 57:1-4.

2. Wang Dapeng. Optimal Design of Payload Cabinet Structure[J]. Spacecraft Engineering, 2010, 19(2):3439.

3. Ding Tao, Peng Wang. Application of HyperMesh Secondary Development in Pre-processing of Bus Finite Element Analysis[J]. Bus Technology and Research, 2015,6:47-49.

4. Jiang Xuewu, Wu Xinyue et al. Comprehensive Application of UG, HyperMesh and MSC Marc in Finite Element Analysis[J]. Computer Aided Engineering, 2007, 16(12) :11-14.

5. Bai Kunxue, Yin Shaoping. Finite Element Analysis for Torpedo Seawater Pipe Based on HyperMesh and Nastran[J]. TORPEDO TECHNOLOGY, 2012, 20(1) :14-18.

6. Yin Mei, Wang Chenglong. Analysis of Brake Disk Modal Optimization Based on HyperMesh and Nastran[J]. Design Research, 2015, 30-33.

7. Faycal Benyahia, Abdulmohsen Albedah. Elliptical and Circular Bonded Composite Repair under Mechanical and Thermal Loading in Aircraft Structures.

8. Clough R W. The finite element method in plane stress analysis[A]. Proceedings of ASCE Conf Electron Computation, Pittsburg, PA, 1960.

9. Courant R. Variational methods for the solution of problems of equilibrium and vibrations[J]. Bulletin of the American Mathematical Society, 1943, 49:123.

10. Turner M J, Clough $\mathrm{R} \mathrm{W}$, Martion $\mathrm{H} \mathrm{C}$,et al. Stiffness and deflection analysis of complex structures[J]. Journal of the Aeronautical Sciences, $1956,23: 805-823$. 